\title{
Neutrophil Contribution in Facilitating Optic Nerve Regeneration
}

\author{
Bhagat Singh* and Jason R. Plemel ${ }^{\star}$ \\ Hotchkiss Brain Institute and Department of Clinical Neurosciences, Faculty of Medicine, University of Calgary, Alberta, Canada, T2N1N4 \\ Review of Kurimoto et al.
}

Promoting long distance axonal regeneration of CNS axons is a lofty goal that has challenged and motivated neuroscientists for decades. CNS regeneration is so challenging because after development neurons lose their intrinsic capacity to regenerate. Even if injured fibers are coaxed to regenerate, growth is hindered by a plethora of inhibitors.

Despite these challenges, regeneration of a small percentage of optic nerve fibers can be induced by ocular inflammation. Investigation of factors secreted by macrophages in ocular inflammation led to the discovery that oncomodulin - a small calciumbinding protein - is a key molecule for optic nerve outgrowth (Yin et al., 2009). However, oncomodulin mRNA expression increases early after injury, peaking within 24 $\mathrm{h}$ of inflammation when macrophage presence is minimal, indicating that cells other than macrophages may be an alternative source of oncomodulin (Yin et al., 2009). Kurimoto and colleagues (2013) provide new evidence that neutrophils are an important component of the ocular inflammatory re-

Received Nov. 18, 2013; revised Dec. 15, 2013; accepted Dec. 17, 2013.

J.P. has fellowship awards from CIHR and the Donna Joan Oxford award from the MS Society of Canada. We thank Michael Keough, Lesley Soril, Anand Krishnan, Shane Eaton, and Wee Yong for their critical appraisal of this article.

The authors have no financial interests or other conflicts of interest to declare.

*B.S. and J.R.P. contributed equally to this work.

Correspondence should be addressed to Bhagat Singh, Hotchkiss Brain institute, Heritage Medical Research Building 165, University of Calgary, 3330 Hospital Drive, NW, Calgary, Alberta, Canada, T2N1N4. E-mail: singhb@ucalgary.ca.

DOI:10.1523/JNEUROSCI.4827-13.2014

Copyright $\odot 2014$ the authors $\quad 0270-6474 / 14 / 341081-02 \$ 15.00 / 0$ sponse that promotes optic nerve regeneration by the secretion of oncomodulin.

One of the earliest responses to injury mounted by the immune system is the infiltration of neutrophils, an important immune cell characterized by its capacity to kill pathogens and phagocytose cellular debris. Neutrophils invade the site of inflammation early after injury, but historically they were thought to be a damaging component of the immune response because they produce and release toxic reactive oxygen species and proteases that cause secondary injury. Recently, cells expressing Gr1-a common marker of neutrophils - were found to drive wound healing, promoting a beneficial response after spinal cord injury (Stirling et al., 2009). These data raise the question: do neutrophils participate in inflammationinduced regeneration?

Kurimoto and colleagues (2013) sought to dissect the role of neutrophils and macrophages in promoting optic nerve regeneration induced by ocular inflammation. To better understand the properties of ocular inflammation, the authors performed cell sorting and immunostaining techniques after zymosan-induced intraocular inflammation and found that during the first $3 \mathrm{~d}$, neutrophils were recruited to a much greater extent than macrophages (Kurimoto et al. 2013, their Fig. 1). Both neutrophils and macrophages that were recruited to the eye expressed oncomodulin mRNA and protein, but with a different temporal pattern of expression. Gr1+ neutrophils highly expressed oncomodulin at 12 and $24 \mathrm{~h}$, but oncomodulin levels decreased thereafter; in contrast, macrophages had low oncomodulin levels at
12 and $24 \mathrm{~h}$ and levels subsequently increased. By $72 \mathrm{~h}$ after inflammation induction, approximately equal numbers of macrophages and neutrophils were recruited into the eye (Kurimoto et al., 2013, their Fig. 2). Thus, the immune response in the eye is well orchestrated, with dynamic involvement of neutrophils and macrophages.

Given that oncomodulin is an essential molecule for inflammation-induced regeneration (Yin et al., 2009) and is expressed almost exclusively by neutrophils within the first $24 \mathrm{~h}$ of inflammation, Kurimoto and colleagues (2013) investigated the impact of neutrophil deletion on inflammationinduced optic nerve regeneration. To test this, Kurimoto and colleagues (2013) crushed the optic nerve and at the same time injected either zymosan intraocularly or zymosan plus antibodies (injected retro-orbitally into the venous sinus and intraperitoneally) to deplete neutrophils. The depletion of neutrophils using a neutralizing antibody significantly decreased neutrophil entry, but did not greatly affect macrophage accumulation in the vitreous chamber of the eye (Kurimoto et al., 2013, their Fig. 3). The reduction in neutrophils was associated with decreased expression of oncomodulin in the retina and subsequently blunted the zymosan-induced axonal regeneration; the total numbers of axons regenerated (up to $500 \mu \mathrm{m}$ from the injury site) were decreased from $\sim 150$ to 50 after depletion of neutrophils (Kurimoto et al., 2013, their Fig. 4). Neutrophils were thus shown to be a major contributor to inflammatory-induced regeneration. To test the role of oncomodulin, the authors administered $\mathrm{P} 1$, a specific peptide antagonist of oncomodulin that is derived 
from the $N$-terminal region of the protein, and found that this significantly blocked regeneration (Kurimoto et al., 2013, their Fig. 5). Other inflammatory cytokines such as ciliary neurotrophic factor (CNTF), leukemia inhibitory factor (LIF), and interleukin 6 (IL-6) were also tested in vitro for their capacity to induce axonal outgrowth; however, only oncomodulin induced a significant twofold rise in axonal outgrowth of retinal ganglion cells (RGCs) (Kurimoto et al., 2013, their Fig. 5). From these studies, the authors concluded that neutrophils secrete oncomodulin and are a major source of inflammationinduced regeneration.

The work by Kurimoto and colleagues (2013) raises other questions related to inflammation-induced regeneration. For example, how is regeneration sustained given that neutrophils are present only for a short period following inflammation? Is it possible that neutrophils and macrophages interact to sustain the prolonged regenerative response induced by inflammation? For example, the numbers of regenerating axons were significantly higher 2 weeks after inflammation-induced regeneration, despite the greatly reduced number of neutrophils in the eye after $3 \mathrm{~d}$ (Kurimoto et al., 2013). In contrast, macrophages recruitment in the eye was prominent $3 \mathrm{~d}$ after injury (Kurimoto et al., 2013, their Figs. 1D, $2 D$ ) and remained elevated to maintain a sufficient inflammatory response for several days to weeks (Yin et al., 2009, their Fig. 2). Macrophages also prominently express and secrete oncomodulin (Yin et al., 2009, their Fig. 2). Indeed, at $24 \mathrm{~h}$ when normalizing to an internal housekeeping gene, macrophages express three times more oncomodulin mRNA than neutrophils; however, at this time point, neutrophils were five times more numerous than macrophages in the eye (Kurimoto et al., 2013, their Fig. 2). In this respect, the collective impact of both neutrophils and macrophages - an earlier robust response by the neutrophils followed by a sustained response by macrophagesmight preserve the chronic regrowth of axons in a hostile CNS environment. Deletion of macrophages alone or in combination with neutrophils at both initial (day 1-3) and later (1-2 weeks) time points following zymosan-induced inflammation will address this question more firmly.

The fact that only a relatively small proportion of RGCs regenerate raises another question: what feature of these RGCs allows them to respond to ocular inflammation and mount a regenerative response? Is there a particular type of RGC that responds to inflammation or oncomodulin? Thirteen types of RGCs have been identified based on their dendritic field size, branching density, and depth of stratification in the inner plexiform layer (Dacey et al., 2003). Watanabe and colleagues (1993) showed that $\alpha$-cells with largest soma size have superior capacity to regenerate compared with all other RGC types, but unfortunately did not examine the responsible mechanisms. Why one RGC type preferentially regenerates is unclear, but it is possible that different types of RGCs express different levels or combinations of receptors for oncomodulin or other unidentified regeneration-inducing factors. In the future, comprehensive analyses of oncomodulin receptor expression and measurements of axonal regeneration following expression of a fluorescent reporter under the control of the oncomodulin receptor promoter in distinct RGC populations will be important in understanding the properties of inflammation-induced RGC regeneration.

Another intriguing question arising from this research is whether the regenerationinducing role of neutrophils is limited to one or more neutrophil subsets. The heterogeneity of neutrophils is becoming apparent (Beyrau et al., 2012). New evidence from cancer research demonstrates the presence of proinflammatory N1 neutrophil subsets and immunoregulatory $\mathrm{N} 2$ neutrophil subsets. While the role of neutrophil subsets after neural injury remains unexplored, it is possible that distinct neutrophil subsets account for the inflammatory-induced regeneration. A greater understanding of the characteristics associated with neutrophilinduced regeneration is warranted and might result in new targets for nerve repair.

Despite the prominence of neutrophils in optic nerve regeneration, neutrophils were found to have no significant impact on peripheral nerve regeneration. Nadeau and colleagues (2011) previously showed that depletion of neutrophils did not affect recovery following sciatic nerve injury. In contrast, Kwon and colleagues (2013) found that escalated recruitment and activation of macrophages adjacent to dorsal root ganglia sensory neurons promoted outgrowth of these neurons. Interestingly, Kwon and colleagues (2013) found that oncomodulin was a prominent component of the macrophageconditioned media responsible for promoting axonal outgrowth. Although the involvement of neutrophils was not investigated in this study, it demonstrates that inflammationand specifically oncomodulin-enhances axonal growth in both the PNS and CNS, in line with the findings by Kurimoto and colleagues (2013).

In summary, Kurimoto and colleagues (2013) demonstrated the importance of neu- trophils_-long considered to be damaging to the cells recruited early after injury-in promoting CNS axonal regeneration. This exciting work leads to many questions for future study, such as what are the molecules downstream of oncomodulin signaling, which neutrophil subsets are involved in inflammation-induced regeneration, and why do only a small portion of RGCs respond to inflammation? Moreover, since neutrophils are known to be cytotoxic (Dinkel et al., 2004), the balance between the beneficial and detrimental properties of neutrophils for nervous system injury will have to be addressed. Given that there is no current therapeutic treatment for spinal cord injury, it is of great importance to understand the intertwined signaling between immune cells and nervous system that promote axonal regeneration.

\section{References}

Beyrau M, Bodkin JV, Nourshargh S (2012) Neutrophil heterogeneity in health and disease: a revitalized avenue in inflammation and immunity. Open Biol 2:120134. CrossRef Medline

Dacey DM, Peterson BB, Robinson FR, Gamlin PD (2003) Fireworks in the primate retina: in vitro photodynamics reveals diverse LGNprojecting ganglion cell types. Neuron 37:1527. CrossRef Medline

Dinkel K, Dhabhar FS, Sapolsky RM (2004) Neurotoxic effects of polymorphonuclear granulocytes on hippocampal primary cultures. Proc Natl Acad Sci U S A 101:331-336. CrossRef Medline

Kurimoto T, Yin Y, Habboub G, Gilbert HY, Li Y, Nakao S, Hafezi-Moghadam A, Benowitz LI (2013) Neutrophils express oncomodulin and promote optic nerve regeneration. J Neurosci 33:14816-14824. CrossRef Medline

Kwon MJ, Kim J, Shin H, Jeong SR, Kang YM, Choi JY, Hwang DH, Kim BG (2013) Contribution of macrophages to enhanced regenerative capacity of dorsal root ganglia sensory neurons by conditioning injury. J Neurosci 33:15095-15108. CrossRef Medline

Nadeau S, Filali M, Zhang J, Kerr BJ, Rivest S, Soulet D, Iwakura Y, de Rivero Vaccari JP, Keane RW, Lacroix S (2011) Functional recovery after peripheral nerve injury is dependent on the proinflammatory cytokines IL-1beta and TNF: implications for neuropathic pain. J Neurosci 31: 12533-12542. CrossRef Medline

Stirling DP, Liu S, Kubes P, Yong VW (2009) Depletion of Ly6G/GR-1 leukocytes after spinal cord injury in mice alters wound healing and worsens neurological outcome. J Neurosci 29:753-764. CrossRef Medline

Watanabe M, Sawai H, Fukuda Y (1993) Number, distribution, and morphology of retinal ganglion cells with axons regenerated into peripheral nerve graft in adult cats. J Neurosci 13:2105-2117. Medline

Yin Y, Cui Q, Gilbert HY, Yang Y, Yang Z, Berlinicke C, Li Z, Zaverucha-do-Valle $\mathrm{C}, \mathrm{He} \mathrm{H}$, Petkova V, Zack DJ, Benowitz LI (2009) Oncomodulin links inflammation to optic nerve regeneration. Proc Natl Acad Sci U S A 106: 19587-19592. CrossRef Medline 